\title{
Uber einige Formen der dritten Person in der lappischen Konjugation
}

\begin{abstract}
]
Über die Herkunft der Formen der 3. Pers. Sg. und PI. im Indikativ Präsens der lappischen Verbalflexion sind verschiedene Deutungen vorgelegt worden, vgl. zuletzt Mrkко КовноNEN MSFOu 143 S. 230-234, 331-337 m. Lit. In den MSFOu 125 S. 92-93 hat der Unterzeichnete kurz die Auffassung vertreten, dass das frühurfinnische Formpaar *kantaja 'der' Träger': *kantajat 'die Träger' Ausgangspunkt war z.B. für die norwegischlappischen Formen 3. Sg. Präs. guod'da 'er trägt' : gud'dik 'sie tragen'. Das alte Suffix des Nomen agentis *ja, * $j \ddot{a}$ ist im Lappischen auch das Zeichen des Partizip Präsens; lpN guod'de (<*kantaja) bedeutet also sowohl 'Träger' als auch 'tragend'. Die Formen der 3. Pers. Sg. und Pl. Präs. haben sich gerade aus dem Partizip Präsens entwickelt. Lautlich entsprechen sie in keinem heutigen Dialekt mehr dem Nominativ Singular und Plural des Partizip Präsens (z.B. lpN guod'de 'tragend, der Tragende': guod'dek 'die Tragenden'). Zu verschiedenen Zeiten vollzog sich in der Struktur entweder der diesbezüglichen Personalformen oder auch des Partizip Präsens eine Sonderentwicklung. Nun kann man fragen, ob nicht in einer derart starken Differenzierung das Bestreben zum Ausdruck kommt, die Vertreter der verschiedenen Funktionen der Deutlichkeit halber auch lautlich voneinander zu trennen. Dieselbe Tendenz hat übrigens darauf einwirken können, dass sich das osfi. Partizip Präsens aufteilte in die Lautgestalten kantava : kantavi. "Lautgesetzliche» Entsprechungen des frühurfi. Typs *kantajat sind nur die Formen der 3. Pl. Präs. im

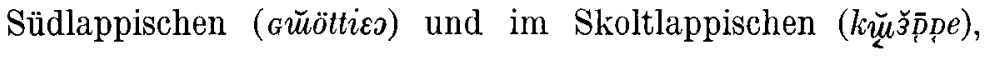


und auch von ihnen mit Bestimmtheit nur die letztgenannte. Nirgends erscheint in der 3. Sg. ein solcher Typus, der auf die Form *kantaja zurückzuführen wäre.

Gemeinsam für alle Dialekte ist in den Formen der 3. Pers. Sg. der gleichsilbigen Verben, dass sie quantitativ mit dem blossen starkstufigen Stamm zusammenfallen, aus welchem Grund E. N. SEтӥL ̈̈ folgerte, dass die 3. Sg. im Lappischen gar keine Endung gehabt habe. Und doch entspricht diese Form hinsichtlich ihres Auslautvokals nicht dem absoluten Stamm bei den $e$-stämmigen (guod'da, boatta 'er kommt' statt *guod'de, *boatte, vgl. Inf. guod'det, boattet) und auch nicht bei den $\hat{a}-$ stämmigen Verben (mânna 'er geht' statt *mânnâ, vgl. Inf. mânnât); bei den $o$-stämmigen Verben entspricht sie dem Stamm im Ostlappischen sowie in den see- und finnmarklappischen Dialekten des Norwegischlappischen (gillljo 'er schreit', orro 'er ist, wohnt', vgl. Inf. gill ljot, orrot), endet aber auf $u$ in der tornelappischen Dialektgruppe des Norwegischlappischen und in allen schwedischlappischen Dialekten (gil'lju, orru).

Vom qualitativen Standpunkt aus könnten die Auslautvokale der Typen guod'da, orru am besten so erklärt werden, dass sie durch Kontraktion des urlp. Stammauslautvokals a bzw. $\omega$ mit dem in der dritten Silbe stehenden engen Vokal entstanden wären. KNUT BERGSLAND hat es denn auch für möglich gehalten, dass diese Formen die Variante *je der Ableitungssilbe ${ }^{*} j a,{ }^{*} j \ddot{a}$ als Suffix hatten, z.B. ${ }^{*} u r o-j e>{ }^{*}$ ŏruje $>*$ orr $\bar{u}>*$ orru $>$ orru, s. Virittäjä 1960 S. 330 . Dann würde der Typus orro natürlich auf die Analogie der 1. und 2. Sg. Präs. zurückgehen (orom, orok). Bergsland geht nicht auf die Frage ein, wann und unter welchen Umständen die in Frage stehende Variante *je entstanden wäre. BJörn Collinder kommt in seiner Erklärung Bergsland nahe. Er führt den Typus guod'da auf die Lautgestalt *kantaji zurück, die sich in früher (vorfinnischer) Zeit aus dem Nomen agentis *kantaja entwickelt hätte. Das $i$ wäre dem $i$ vergleichbar, das in dem fi. Wort kumpi ( liumpa-) 'welcher von beiden' erscheint, s. Comparative Grammar 306. Wie Bergsland feststellt, ist der Typus mânna, gulla 'er hört' auf jeden Fall eine Analogie zum Typus 
boatta (a.a.0. 329). Collinder vermutet zwar, dass sich das $i$ der zweiten und dritten Silbe der Urform *meniji im Lappischen zu einem dunklen $\hat{a}$ entwickelt hätte; die Kontraktion hätte dann ein helles $a$ ergeben. Dies ist jedoch nicht möglich, denn ${ }^{*} \hat{a}+{ }^{*} \hat{a}$ (frühurlp. ${ }^{*} e+{ }^{*} e$ ) hätten nur $i$ ergeben, vgl. Verf., MSFOu 79 S. 343, Bergsliand a.a.().

Eine relativ alte Veränderung eines auslautenden $a, \ddot{a}$ zu $i$ hat im Ostseefinnischen nur in zwei Formgruppen nachgewiesen werden können, nämlich in der 3. Sg. Präs. (und Pot.) sowie im Nominativ des Komparativs, z.B. fi. saapi 'er bekommt', kantavi 'er trägt' (vgl. 3. Pers. Pl. Präs. saavat, kantavat), kumpi 'welcher von beiden', suurempi 'grösser' (vgl. Nom. Pl. kummat, suuremmat). Der Wandel $a, \ddot{a}>i$ der Personalendung der 3 . Sg. und des Komparativsuffixes hatte als Zwischenstufe vielleicht ein ${ }^{*} e$, also z.B. ${ }^{*}$ säpe $>$ sãpi wie z.B. Nom. Sg. *lapse >lapsi 'Kind'. Die Verengung eines offenen Vokals zu ${ }^{*} e$ in der $3 . \mathrm{Sg}$. Präs. dürfte eine Reduktionserscheinung sein, verursacht durch schnelles Sprechtempo, hohe Frequenz der diesbezüglichen Form sowie vielleicht auch (s. oben S. 98) das Streben nach Vermeidung von Homonymie. Die entsprechende Veränderung des Komparativzeichens lässt sich schwerer verstehen, falls nicht das Sprachgefühl den zweiten Teil des Suffixes $m p a$, mpä verbunden hat mit der Endung des Partizip Präsens, wobei es mit der Veränderung des Nominalsatzes vom Typ *mies menevä zu mies menevi'der Mann geht' auch zu der Neuerung *mies suurempa $>$ mies suurempi 'der Mann (ist) grösser' kam. Später hätte sich dann $i$ auch in der attributiv verwendeten Komparativform durchgesetzt.

Recht üblich dürfte jedoch die Auffassung sein, dass der Wandel von auslautendem $a, \ddot{a} \mathrm{zu} i$ im Urfinnischen auch anderwärts hätte eintreten können, und zwar in der zweiten Silbe nach einer langen ersten Silbe und in der dritten Silbe unabhängig von der Struktur des Wortstammes. Infolge analoger Ausgleichung, d.h. indem sich das inlautende $a, \ddot{a}$ im gesamten Substantivparadigma einbürgerte, wären die Zeichen einer diesbezüglichen Veränderung im Ostseefinnischen späterhin fast spurlos verschwunden. Diese Annahme beruht in erster Linie 
auf der von Heikk I OJansud in JSFOu XXX,17 S. 13-16 veröffentlichten Studie, wo aufgrund von Beobachtungen im Feld eine bestimmte Lauterscheinung im Südestnischen behandelt wird. Wäre Ojansuus Erklärung richtig, würde sie die oben angeführte Hypothese tatsächlich unbestreitbar rechtfertigen. Da die Frage für die ostseefinnische - und sogar auch für die lappische - Vokalgeschichte wichtig ist, soll sie hier exkursmässig behandelt werden. Verf. muss zwar zugeben, dass er die umfangreiche Materialsammlung von Ojansuu, die allerdings nur wenig morphologische Aufzeichnungen enthält, nicht so gründlich durchgehen konnte, wie es für diesen Zweck empfehlenswert gewesen wäre.

Nach Ojansuu ist im Osten des Dialektgebietes von Võrumaa, bei den Setukesen und den Esten von Lutsi und Kraasna der Auslautkonsonant $m, n, l, r, s, v$ im apokopierten Nominativ Sg. der dreisilbigen $a$ - und $\ddot{a}$-stämmigen Nomina ganz allgemein mouilliert, z.B. an̆nom (Gen. anòmà) 'Gefäss', păkkan' (Gen. pagànà) 'Heide', inūmal (Gen. ìumàsà) 'Gott', maìtal (Gen. madàsà) 'niedrig', kevvvegr (Gen. kevèrà) 'falsch, betrügerisch' ( fi. kovera 'ausgehöhlt'), aher' (Gen. ahtrà) 'aufgetrocknet, gelt (Kuh)' ( fi. ahtera id.), i.̌.soś (Gen. isòzà) 'schön' ( fi. iloisa 'fröhlich'), verrev' (Gen. verèvä) 'blutvoll, rot', vä̌̆kkev' (Gen. vägèvẫ) 'stark'. Er führt sie auf die urfi. Formen *jumali, ${ }^{*}$ pakani, ${ }^{*}$ väkevi usw. zurück und vermutet gleichzeitig, dass der Wandel des auslautenden $a, \ddot{a}$ zu $i$ zumindest in dreisilbigen Wörtern im Urfinnischen eine regelmässige Erscheinung gewesen wäre.

In den meisten südestnischen Dialekten begegnet die diesbezügliche Mouillierung nach den Aufzeichnungen von Ojansuu nicht. In den Dialekten von Puhja, Võnnu, Rõngu, Kambja, Otepää und Sangaste in Tartumaa sowie in den Dialekten von Hargla, Karula, Urvaste, Kanepi, Põlva und Räpina in Võrumaa scheint die Vertretung ohne Mouillierung zu herrschen: jŭmmal, kav̌val 'schlau, listig', maital (maìal), rum̌mal 'Tölpel'; kevveer (keverer), utar ( $\mathrm{Pl}$. uDara') 'Euter'; orrav (orav) 'Eichhörnchen', pallav 'heiss', saiav (Pl. sadava') 'Wasserfall', sǚkkäv 'tief', ter̆räv 'scharf', veřrev, vä̌̆kkev; hap̀en (Pl. habèna') 'Bart', up̀in 'Kartoffel', värrin 'Beben, Zittern' usw. Dennoch ist z.B. aus Võnnu neben der Form jummal auch der Wunsch jummalappi mit $l$ aufgezeichnet. In einigen südlichsten Dialekten von Võrumaa ist die Mouillierung schon allgemeiner. Ojansuu schreibt, dass im Dialekt von Rõuge $l$ und $r$ mouilliert begegnen, nicht aber $m, n$ und $v$. So hat er auch von dort u.a. à̀ar 'geräumig', maittal, pinnär 'Ackerrain' (vgl. estn. Normalspr. peenar, Gen. peen(d)ra) aufgezeichnet, daneben jedoch 
auch nichtmouillierte Formen: jum̌mal, vetel 'flüssig', kè̀er u.a. Im Dialekt von Vastseliina scheint die mouillierte Vertretung

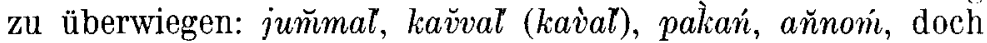
ist auch das Fehlen der Mouillierung keine Seltenheit: aher (Pl. ahtra'), kètar 'Strebe', kum̄mer (Pl. kumera') 'ausgehöhlt', kašsuv 'Gewächs' ( fi. kasvava, dial. kasuva 'wachsend'). Die Esten von Lutsi sind nach der Auffassung von Ojansuu Umsiedler, die Anfang des 18. Jh. aus dem Kirchspiel Vastseliina kamen (s. Iso Tietosanakirja 5, 1913, Spalten 1277-1278). Aus ihrem Dialekt hat Ojansuu u.a. i.r.sos', jumal', otav' 'billig', u'taŕ, vas̀aŕ (Komit. vazaraga) 'Hammer' angeführt; zumindest aufgrund der Vertretung der estnischen Normalsprache müssen auch folgende Wörter dieses Dialekts zu den ursprünglichen $a$-, ä-Stämmen gerechnet werden: kämel (Pl. käàmle ) 'die flache Hand', tungel (Pl. tungle') 'Brand' und väster (Pl. västre') 'Fischgabel' (im Dialekt von Lutsi ist in der urspr. dritten Silbe offenbar in grossem Umfang eine Veränderung von $a, \ddot{a} \mathrm{zu} e$ vor sich gegangen). Andererseits ist bei zahlreichen Fällen keine Mouillierung bezeichnet: kalkun (Pl. kalkkuna') 'Truthahn', keiar, kird'av 'bunt', küp̀är (Pl. kübärä') 'Mütze', lịhav 'fett', 'ớrau, pă̌лav (palav), up̀in (Pl. uвina'), verrreu (v́errev, vereu). Das eigentliche Beleggebiet der Mouillierung des Auslautkonsonanten im Nominativ der $a$-, $\ddot{a}$-Stämme ist der setukesische Dialekt, wo die Erscheinung so allgemein ist, dass irgendein einzelnes Beispiel, wo sie nicht bezeichnet ist (z.B. matal), wie ein Schreibfehler wirkt. Z.B. ap̆paŕ (Pl. abara'), hirmmus' 'schrecklich', iл̌soś, jum̃mal, kị̆peń (Pl. kịbena') 'Funke', kù.лuś 'be-

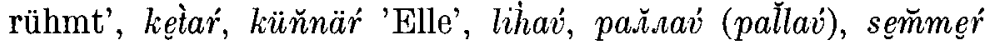
(Elat. Sg. semèrast) 'Kies', up̆pin (Pl. ubina'), vajus' 'licht, hell', vašsaŕ (vas̀aŕ), väkè́. Da die Esten von Kraasna ursprünglich Setukesen von Petseri sind, die wahrscheinlich in der zweiten Hälfte des 16. Jh. aus ihrer Heimat kamen (vgl. O. Kallas Kraasna maarahvas $46-54$ ), finden wir die von uns untersuchte Erscheinung der Mouillierung natürlich auch hier, z.B. jumal (Kallas, a.a.0. 78, 79 usw.), vassar (a.a.O. 25), sipul (a.a.O. 124) 'Zwiebel' (wenn nämlich dies $<{ }^{*}$ sipula; vgl. auch Vastseliina sipul, Nom. Pl. sibula). Ohne Zeichen der Mouillierung sind z.B. habõn (a.a.O. 124), illoz (a.a.O. 125), küpär (a.a.O. 78), upin (a.a.O. 45), valuz (a.a.O. 125) geschrieben; zu der Zuverlässigkeit dieser Schreibweisen kann Verf. nichts sagen.

Wenn auch Ojansuus Erklärung der Herkunft des südestnischen Typs jummal in verschiedenen Zusammenhängen wiederholt worden ist, kann sie dennoch Zweifel erwecken. Zunächst lässt sich einmal feststellen, dass vom Worte sezar (Helme, 
Sangaste), sizar (Lutsi) 'Schwester', dessen Nominativ ursprünglich zweisilbig ist (würde es sich um eine apokopierte Form handeln, hiesse sie ${ }^{*}$ sešsar, vgl. vašsar $<{ }^{*}$ vasara), auch eine auf $\dot{r}$ endende Form verbreitet auftritt: sezar (Puhja, Rõuge), sizaŕ (Petseri, Lutsi). Strukturmässig steht diesem Wort tüttär (Võ̃nnu) 'Tochter' nahe, das häufiger auf $\dot{r}$ endet: tütär' (Rõuge, Petseri), tüttär (Otepää, Petseri). Der mouillierte Auslautkonsonant dieser Wörter wird durch Ojansuus Theorie als solche nicht erklärt, denn wenn ihr Nominativ im Südestnischen früher stellenweise auf $i$ endete, kann dieser Vokal nicht urfinnisch sein. Das Wort der estnischen Normalsprache sõsar hat einen auf $a$ endenden Vokalstamm (z.B. Gen. sõsara), der natürlich sekundär ist. Denselben Stand weist z.B. der Dialekt von Rõuge auf (Nom. Pl. sezara). Die Frage, wie die auf $e$ bzw. $e$ auslautenden Vokalstämme einiger anderer Dialekte zu beurteilen sind (z.B. Sangaste Nom. Pl. sezare; in diesem Dialekt begegnet in der 3 . Silbe der Wandel $a, \ddot{a}>e$, vgl. KetTunen EKÄH 152; Põlva sizare ${ }^{c}$, Petseri sizare ${ }^{c}$ ), muss der Unterzeichnete offen lassen. In den Volksliedsammlungen Ojansuus begegnen denn auch tatsächlich die auf $i$ endenden singularischen Nominative sizari (Põlva, Petseri), tütteri, tüieri (Petseri), mit denen die in Petseri aufgezeichnete Form madali 'niedrig' aus der Sprache der Volksdichtung vergleichbar ist. Eventuell haben wir hier durch das Versmass erhaltene Archaismen, wie sie in der estnischen Volksdichtung bekanntlich häufig vorkommen. Andererseits erhebt sich die Frage, ob wir es hier nur mit Analogieformen zu tun haben, entstanden aus der Notwendigkeit des Versmasses; z.B. pikkem̌bi $\sim$ pi ǩkeb' 'länger', keneli $\sim$ kenel 'er sprach' in der Sprache der Volksdichtung hätten dann als Muster dienen können. Der Vokal der 2. Silbe in der Form tüteri von Petseri ist natürlich sekundär, vgl. tütäreni 'meine Tochter', das in einem Lied aus Karula vorkommt. Wenn wir annehmen, dass die in Frage stehenden Nominative auf $i$ seinerzeit wirklich auch zur gesprochenen Sprache bestimmter Dialekte gehört hätten, so würden sie sich am natürlichsten als Abstraktionen aus den Pluralstämmen erklären, vgl. z.B. Pôlva Allat. Pl. sizarile. Sie hätten aber trotzdem nicht die alleinige Vertretung bilden können, sondern wären nur eine Art Nebenformen von ursprünglich zweisilbigen Nominativen. Ein ${ }^{*}$ sesari z.B. wäre nämlich apokopiert zu *sešsar geworden, so dass die Form sezar eine Kontamination der Typen *sesar und *sesari sein müsste.

Auch der Nominativ Sg. vom Typ madali statt ${ }^{*}$ madala könnte auf der Verallgemeinerung des Pluralstammes beruhen, 
da nach den Aufzeichnungen von Ojansuu zahlreiche Dialekte in den dreisilbigen $a$ - und $\ddot{a}$-Stämmen einen $i$-Plural kennen, z.B. Karula Gen. Pl. keveride, Rõuge Gen. Pl. jumalide, Komit. Pl. jumalidega, Partit. Pl. jumalit, Transl.Pl. jumalis, Elat. Pl. jumalist, Vastseliina Gen. Pl. keveride, Lutsi Allat. Pl. jumaлi.лe, Elat. Pl. ubinist, Petseri Illat. Pl. jumasihi, Partit. Pl. menebit 'beide', nä̈dälit 'Woche'. Das Auftreten des Typs madali im setukesischen Dialekt und den daran grenzenden Mundarten von Võrumaa neben dem Nom. Sg. auf ${ }^{*} a$ oder statt dessen wäre eine Angleichung an die urspr. singularischen Nominative auf $* i$, die sowohl in den eigenständigen als besonders in den entlehnten Wörtern zahlreich vertreten sind. Es sei an Ojansuus Beobachtung erinnert, dass in Rõuge nur der Konsonant der Endsilben ${ }^{*} l a\left({ }^{*} l \ddot{a}\right),{ }^{*} r a\left({ }^{*} r a ̈\right)$ mouilliert ist. Ist es ein blosser Zufall, dass bei den dreisilbigen $i$-stämmigen Nomina gerade die auf ${ }^{*} l i,{ }^{*} r i$ auslautenden eindeutig in der Mehrzahl zu sein scheinen? Hierher gehören u.a. folgende Wörter: kappel (Võnnu), kasel' (Gen. kaseli; Puhja) '(Friedhofs)kapelle', kurel' (Rõngu) 'Kurier', kühvel (Helme) 'Schaufel', pelkel (Lutsi) 'Stampfer, Stössel', potel (Hargla) 'Flasche', retel (Nom. Pl. redeli', redeli; Lutsi) 'Leiter', rö̀vel (Sangaste) 'Räuber'; uhmeŕ (Nom. Pl. uhmri'; Sangaste), huhmeŕ (Pl. huhmrę; Karula), uhmer (Kanepi), u'mer (Lutsi) 'Mörser', kammer (Hargla) 'Kammer', kutsaŕ (Otepää, Karula) 'Kutscher', köster (Puhja), köster (Rõngu) 'Küster', pap̌peŕ, pà̀eŕr (Petseri) 'Papier', vikur (Nom. Pl. viguri; Urvaste, Petseri), viguŕ (Põlva) 'störrisch, launisch'. Von ihnen aus hätte sich der auf $i$ endende Nominativ in jene $a$-, $\ddot{a}$-stämmigen Wörter verbreiten können, vor deren Auslautvokal ein $l, r$ stand. Die Vermischung der Stammtypen konnte ferner intensiv dadurch gefördert werden, dass zumindest der Genitiv und Komitativ Pl. bei den dreisilbigen $a(\ddot{a})$ - und $i$-stämmigen Wörtern von jeher lautlich zusammengefallen sein dürften. Hier einige Beispiele für $i$-Stämme: Gen. Pl. rüblide 'Rubel', Komit. Pl. pek̀linega 'mit den Stampfern' (Petseri); vgl. damit die oben erwähnten Formen jumalide, jumalidega. Nach den Angaben von F. J. Wiedemans zu schliessen, könnte die Übereinstimmung zwischen den Worttypen jummal und rövel sogar für die ganze Pluralflexion gelten. In seinem »Versuch über den werroehstnischen Dialekt" S. 29 sagt Wiedemann zuerst, dass in diesem Dialekt statt des Nom. Sg. vom Typus höwel 'Hobel' auch die Form hồwli vorkommt, »indem man es vorzieht, die vocalisch auslautende Form des Genitivs schon im Nominativ zu gebrauchen, dessen eigentliche Form zum Theil und mundartlich wohl daneben gehört wird". Zu derselben "Klasse" wie hồwli zählt er u.a. die Wörter pêkri 'Becher' (vgl. Nom. Sg. 
peeker, Gen. Sg. peekri in der Gemeinsprache), rūbli 'Rubel' und rồwli 'Räuber', deren Pluralflexion dem Schema Partit. pêkrit, Gen. pêkride, Elat. pêkrist folgen soll. Aufgrund einer gemeinsamen Pluralflexion hätten die mouillierten Auslautkonsonanten leicht auch in den apokopierten Nominativ der $a(\ddot{a})$-Stämme verallgemeinert werden können, ohne dass dabei ein analoger Nominativ auf $i$ als notwendige Zwischenstufe vermutet werden müsste.

Für die gegenseitige Beeinflussung der $a(\ddot{a})$ - und $i$-Stämme wird ferner zeugen, dass nach den Aufzeichnungen von Ojansuu zu gehen im Nom. Sg. der auf *ri endenden Nomina sehr häufig ein $r>r$-Wandel eingetreten ist. Bestimmte Wörter können sogar in dem einen Dialekt $a$-stämmig, im anderen $i$-stämmig sein, z.B. Gen. Sing. kańepi (Urvaste) kańeba (Lutsi) 'Hanf', Partit. Sing. pudelit (Hargla) pudesat (Petseri) 'Flasche'.

Es sei noch hinzugefügt, dass das analoge Auslaut-ń unbestreitbar zumindest im Nom. Sg. zweier Deminutiva in den Dialekten von Vastseliina, Lutsi und Petseri begegnet: Vastseliina hoperi, Petseri hopppeń (Gen. Sg. hobeze; in der Volks-

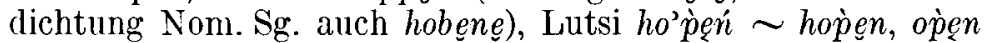
(Gen. Sg. hobeze) 'Pferd', vgl. Helme ове̨nę, Põlva (Volksd.)

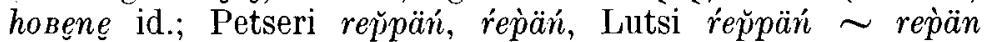
(Nom. Pl. rebäze') 'Fuchs'. Diese Formen hatten wohl in keiner Phase einen Vorgänger auf $i$, sondern sie dürften auf der Analogie von hap̆peńn (< hay̆pen) u.ä. Fällen beruhen. Setukesische dialektale Nominative wie I v̌vań, Iv̀an (Gen. Ivana; $<$ russ. Иван) und karmaí 'Tasche' (< russ. карман) sind ein Beweis für die Stärke der Verallgemeinerungstendenz des Auslaut- $\dot{n}$ und unterstützen gleichzeitig die Auffassung, dass die Mouillierung des Auslautkonsonanten im Nominativ der dreisilbigen $a-, \ddot{a}$-stämmigen Nomina eine späte, vor allem im setukesischen Dialekt entstandene Erscheinung ist. ${ }^{1}$

1 In einem späteren Aufsatz (Suomalaisen Tiedeakatemian esitelmät ja pöytäkirjat 1920 S. $61-73$ ) hat OjANsuU seine Erklärung betreffs des südestnischen Typs maťtàl dahingehend geändert, dass dieser sich aus * matali durch die Vermittlung der schon im Urfinnischen vorhandenen Zwischenstufe *matail entwickelt habe. Das Suffix ${ }^{*} m a\left({ }^{*} m \ddot{a}\right)$ hätte in mehreren Fällen eine ähnliche "Metathesis" * $m a\left({ }^{*} m \ddot{a}\right)>{ }^{*} m i$ $>{ }^{*}$ im durchgemacht, was u.a. das Suffix in $(\sim$ ime $)$ der Nomina instrumenti (z.B. *kolema $>{ }^{*}$ kólemi $>{ }^{*}$ kōleim $>{ }^{*}$ kōlein $>*^{*}$ kōlin $>$ fi. kuolin- in kuolinpäivä 'Sterbetag' usw.) und der Superlativcharakter in ( im Urfinnischen *ima) zeigen würden. Wahrscheinlich beruhen jedoch die von Ojansuu behandelten verwickelten paradigmatischen Verhältnisse, in denen schon er selbst vielerlei analogische Ausgleichungen bemerkt, darauf, dass mit den Suffixen ${ }^{*} m\left(\sim{ }^{*} m e\right)$ und ${ }^{*} m a\left({ }^{*} m a ̈\right)$ abgeleitete Wörter Mischparadigmen verschiedenen Typs gebildet haben, 
Als Anhänger der Theorie Ojansuus sah Lauri Kettunex in der Mouillierung des Auslautkonsonanten solcher livischen Nominative wie ovàr 'breit' ( $<$ *avara) und pagàn' 'Heide' $\left(<{ }^{*}\right.$ pakana) ebenfalls eine Folge des urfinnischen Wandels $a>i$, s. Livisches Wörterbuch S. LIII. Anders und richtig hat Lauri Postr diese Fälle erklärt: "Der palatalisierte Konsonant stammt aus dem Plural: Part. Pl. ovàrí, kảigarí, pagànii", s. MSFOu 85 S. 286. Später meinte auch Kettunen, dass Postis Auffassung möglicherweise richtig sei, liess sie jedoch nur alternativ neben der früher von ihm gegebenen Erklärung gelten, s. MSFOu 119 S. 194.

Die ostseefi. Suffixe des Partizip Präsens und des Komparativs haben auch im Lappischen Entsprechungen, und gerade in diesen Suffixen würden wir ja einen Beweis für den von Collinder angenommenen finnisch-lappischen Wandel $a, \ddot{a}>e(i)$ erwarten, doch den sucht man vergebens, z.B. nub'be, mub be (nicht: *mub'bâ) 'ein anderer' $\left(<^{*} m \bar{u}-m p a\right)$, guod'dep (nicht: *guod'dap) 'wir tragen' ( $<{ }^{*} k a n t a p a$ bzw. *- $\left.\beta a\right)$. Man kan wie Korhonen auch darauf hinwcisen, dass es im Lappischen auch viele andere Konjugations- und Deklinationsformen gibt, die die Voraussetzungen für die genannte Veränderung gehabt hätten, in denen diese Entwicklıng aber nicht eingetreten ist, s. a.a.0. 231 .

Letzten Endes scheint die Diskussion darüber, wann die Variante *kantaje $\left({ }^{*}\right.$ kantaji) neben der Form kantaja erschienen sei, recht überflüssig, denn die russischlappischen Dialekte beweisen, dass es eine solche Form im Urlappischen gar nicht gegeben hat. Im Russischlappischen begegnet nämlich in etwa zwanzig Formgruppen ein urlappischer oder zumindestens relativ alter Kontraktionsvokal in der zweiten Silbe (vgl. Verf., MSFOu 88 S. 187-192, 244-246), darunter findet sich jedoch kein einziger, wo die Kontraktion vor der offenen letzten Silbe

wobei sich das dem suffixalen $m$ vorangehende $i$ durch Sekretion von solchen Stellungen aus verbreitet hat, in denen es zum Wortstamm gehörte, z.B. leipoi- 'backen': leipoime-, tarpoi- 'mit der Störstange schlagen': tarpoime-, vesoi- 'Sprösslinge abhauen': vesoime-, viskoi-'werfen, worfeln': viskoime-; pohti- 'schwingen': pohtime-, oyyhti- 'haspeln': oyyhtime- (> fi. oyyhdin in oyyhdinpuut'Haspel'); isoi 'gross': *isoima 'grösst', ali- (Ableitung aus ala 'das Untere'): alima(inen), yli- (Ableitung aus ylä 'das Obere'): ylimä(inen), peri- (Ableitung aus perä 'das Hintere'): peri$m a ̈($ inen); zu letztgenannten vgl. das Vorkommen des suffixalen $u \mathrm{im}$ Estnischen in Fällen wie tagupäri, -pidi 'rücklings': tagumine 'hinterst'. 
einer urspr. dreisilbigen Form erfolgt wäre. Gerade eine solche

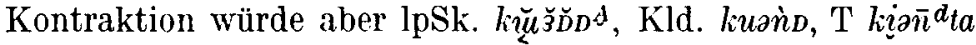
'er trägt' voraussetzen, wenn es eine Kontraktionsform wäre. In quantitativer Hinsicht unterscheiden sich die nichtkontrahierten und die kontrahierten Formen sowohl im Skoltlappischen (vgl. Korhonen, a.a.O. 335) als auch im Kildinlappischen deutlich voneinander; diese Zweiteilung ist völlig konsequent und kennt keine Ausnahme. Den norwegischlappischen Formen mânna 'er geht', boatta 'er kommt', bosso 'er bläst', oaž' žo 'er bekommt' entsprechen im skoltlappischen Dialekt von Pet-

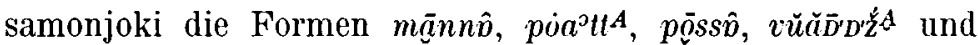
im kildinlappischen Dialekt von Šonguj die Formen man̂, poast, pn $\hat{s}, w_{\text {uañóź }}$. Wären diese Formen kontrahiert, lauteten

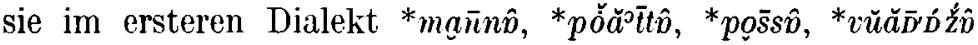
und im letzteren *mā̄na, *poasta, *pns̄sa, *wuañóža (bzw. ${ }^{*}$ mañn $\varepsilon,{ }^{*}$ poadt $\varepsilon$ usw.). Es sei darauf hingewiesen, dass im Dialekt von Šonguj der auslautende nichtkontrahierte Vokal auch in den Wörtern geschwunden ist, deren zweite Silbe früher geschlossen war, während der kontrahierte Vokal stets

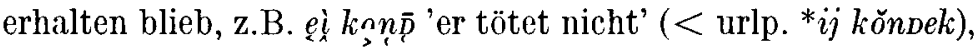

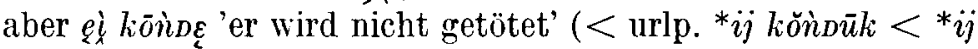

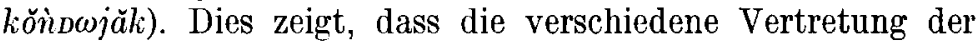
nichtkontrahierten und kontrahierten Vokale ausschliesslich auf jenen ursprünglichen Quantitätsunterschieden beruht, die im Inarilappischen z.B. für diese erwähnten Formen genau entsprechend den späturlappischen Verhältnissen erhalten

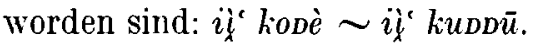

In einem vokalisch auslautenden urspr. dreisilbigen Wort mit einem $* j$ zwischen der zweiten und dritten silbe hat im Russischlappischen nie eine Kontraktion stattgefunden, sondern immer eine Apokope. Vgl. z.B. die Adjektivableitung, die Fülle und Menge bedeutet: lpSk. (Petsamonjoki) Nom. Sg.

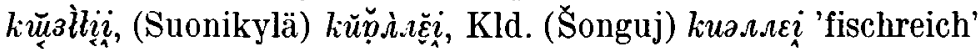
( kontrahierte Formen z.B. im Inarilappischen: kŭăllä und im Lulelappischen: kŭöllä) $<$ urlp. *kolajă (über dieses ${ }^{*} j a ̆$ Suffix genauer s. Verf., Congressus internationalis fennougristarum Budapestini habitus 247-249); 3. Sg. Präs. der kontrahierten Verben: Sk. (Pe) kờttsăi, (Snk.) knŏtsṣ̌i, Kld. 
$k^{\wedge} \dot{D} t s \varepsilon i$ 'er erwacht' ( kontrahierte Formen I kottsā, N

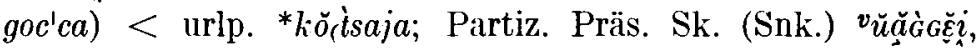

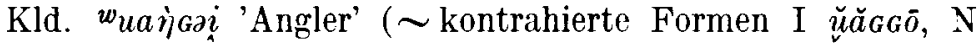
oag'go) $<$ urlp. * ${ }^{*} \dot{\eta}$ g $\omega j a$.

So lässt sich also mit Hilfe verschiedener lautlicher Kriterien des Russischlappischen nachweisen, dass das Präsens der 3. P. Sg. mânna, guod'da, orro (orru) usw. keine kontrahierten sondern urspr. nichtkontrahierte zweisilbige Formen sind.

Hinsichtlich der 3. Pl. Präs. muss aufgrund der Verhältnisse im Süd-, Inari- und Skoltlappischen gefolgert werden, dass ${ }^{*}$ jat, ${ }^{*}$ jät die ursprüngliche Lautgestalt war, vgl. MSFO॥ 79 S. 328, Bergsland, a.a.O. 328, Korhonen, a.a.O. 335. Bei den $a$ - und $e$-stämmigen Verben entstand durch Kontraktion des Auslautvokals des Stammes und des Vokals der Endung urlp. ${ }^{*} \bar{e}\left({ }^{*} \varepsilon\right)$, bei den $o$-stämmigren Verben entsprechend urlp. ${ }^{*} \bar{\omega}$. Die Kontraktionsvokale der genannten Dialekte gehen auf

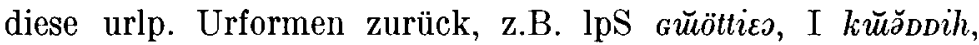

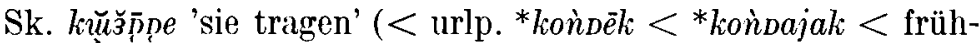

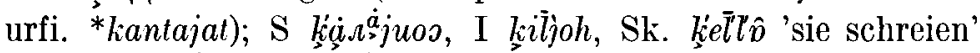

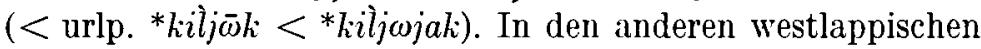
Dialekten ausser dem Südlappischen trat im Vokal der Endung ein sekundärer Wandel ${ }^{*} e>i,{ }^{*} \omega>u$ ein, z.B. N gud'dik, gil'ljuk < * guod'dek, *gil'ljok.

Auch Collinder billigt als Urform der Endung *jat für jene Dialekte, in denen als Kontraktionsvokal ein Vertreter von urlp. $e$ (bzw. $\omega$ ) begegnet; in den Dialekten aber, die ein $i$ (bzw. $u$ ) haben, müsste von der Lautgestalt *jil (*jet) ausgegangen werden; z.B. IpN mânnik < $<$ menijit. Der urspr. Typus *kantajat hätte durch den Einfluss der oben genannten Singularform *kantaji die Lautgestalt ${ }^{*}$ kantajit (*kantajet) erhalten, s. a.a.O. Zu dieser Auslegung ist zu sagen, dass die Entwicklung *menijiı $>$ mânnik zwar vollauf möglich gewesen wäre, dass die Urform *kantajit aber nicht gud'dik sondern *guod'dal ergeben hätte; doch könnte gud'dik auf der Analogie des Typs mânnik beruhen. Aber da wir also die Rekonstruktion *kantaji nicht als motiviert ansehen können, trifft dasselbe natürlich auch für die angenommene pluralische Form ${ }^{*}$ kantajit ( ${ }^{*}$ kantajet) zul. 
Das Inarilappische (vgl. MSFOu 88 S. 118-119) und die westlappischen Dialekte, mit Ausnahme vielleicht des Südlappischen, haben gemeinsam an einer sekundären Entwicklung teilgenommen, deren Resultat die quantitative Angleichung der Formen 3. Pl. Präs. an die urspr. zweisilbigen starkstufigen Formen war, z.B. I maǹèh 'sie gehen', puat'ih 'sie kommen', or̀̀h 'sie sind'. Diese eigenartige Kurzform dürfte in erster I inie den Einfluss der schon früher zweisilbig gewordenen Form der 3. Sg. Präs. (I mañà, puat'á, ờò) widerspiegeln. In dem weit verbreiteten westlappischen Typ mânnik, böttik, orruk ging die Kürzungstendenz noch weiter, indem das $e, \omega$ der zweiten Silbe zu $i, u$ wurde. An den genannten Vokalen vollzog sich also folgende Entwicklung: urlappischer langer kontrahierter Vokal ${ }^{*} \bar{e}\left({ }^{*} \bar{\varepsilon}\right),{ }^{*} \overline{(i)}>\grave{e}, \dot{\omega}$ (das Inarilappische blieb bei dieser Vertretung) $>i, u$. Wir gehen sicher nicht fehl in der Annahme, dass diese Veränderungsprozesse auf jenen Faktoren beruhen, von denen oben im Zusammenhang mit dem Typus fi. kantavi die Rede war. Die regelmässig entwickelten Kontraktionsformen weisen in den pite-, lule- und norwegischlappischen Dialekten die sog. Überdehnung auf, es gibt aber ausser der 3. Pl. Präs. eine ganze Reihe anderer Formen der Verbalflexion, wo sie fehlt, z.B. N 1. Sg. Imperf. bōttim 'ich kam', 2. Sg. Imperf. bōttik 'du kamst', 3. Pl. Imperf. bötti 'sie kamen'. Zweifellos sind auch diese Kurzformen durch die im Leben der Sprache herrschende Ökonomie zustande gekommen. Wie eingangs bereits erwähnt wurde, hat das Skoltlappische (und eventuell auch das Südlappische) in der 3. Person Pl. den archaistischen Typ bewahrt: mạnñe 'sie gehen', pọă kommen', n $\bar{r} r \hat{b}$ 'sie sind' usw. gehen direkt auf die späturlp. Ausgangsformen ${ }^{*}$ măǹe $k\left(<{ }^{*}\right.$ măñăjak < frühurfi. *menejät), ${ }^{*} p \omega \grave{t} \bar{e} k$, *ŏr $\bar{\omega} k$ usw. zurück. (Zum Kildin- und Terlappischen können wir in diesem Zusammenhang nichts sagen, da die in Frage stehende Form in diesen Dialekten geschwunden ist; die 3. P.Du. wird auch in pluralischer Funktion verwendet.)

In den meisten fiu. Sprachen ist die $3 . \mathrm{Pl}$. Präs. aus der 3. Sg. Präs. durch Anhängung des Pluralzeichens gebildet. Z.B. fi. kantavi, kantaa < kantava 'er trägt': kantara-t 'sie tragen', mordw. kandị 'er trägt, bringt': kandị-t' 'sie tragen, bringen', 
tscher. (in der em-Konjugation:) kondà 'er bringt': konda--t 'sie bringen', (Rudiment der urspr. Vertretung in der amKonjugation:) $\grave{u} \cdot l \hat{\imath}$ 'es gibt: $\grave{u} \cdot l \hat{\partial}-t$ 'sie sind', syrj. mune 'er geht', munas 'er geht, wird gehen': mune-ni 'sie gehen', munas-

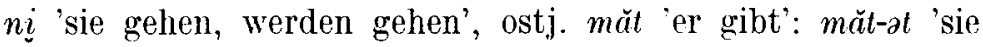
geben', wog. $t \bar{e} \gamma$ 'er isst': $t \bar{e} \gamma-t$ 'sie essen', so vielleicht ursprünglich auch im Ungarischen (vgl. J. SzInNyei FUS'2 131, FUF V 69-70): leszen 'er wird': leszn-eh: 'sie werden'.

In diesem Lichte scheint offenbar zu sein, dass die ursprüngliche Vertretung in jenen wenigen Fällen, wo das lautliche Verhältnis der Formen der 3. Sg. und Pl. nicht dem allgemeinen Schema folgt, auf die eine oder andere Weise gestört ist. Das in der tscheremissischen am-Konjugation herrschende Ver-

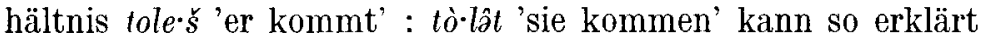
werden, dass im Singular die frühere Form *tò lâ verdrängt wurde durch eine auf $k$ šs $(\partial)$ endende Variante des Nomen agentis, vgl. MSFOu 125 S. 101, 106-108 m.Lit. Im Wotjakischen ist die 3. Sg. Präs. vom Typ baśte 'er nimmt' urpermisch, während die 3. Pl. baśto 'sie nehmen' zweifellos erst spät entstand und - wie T. E. Uotila seinerzeit in seinen Vorlesungen vermutete - das blosse Verbalnomen 'nehmend' sein kann, ohne jegliche Personalendung (zum o-Suffix im Wotjakischen s. MaGDA A. Kövesi A permi nyelvek ösi képzői 39-64 m.Lit.).

Das Verhältnis der dritten Personen im Lappischen untereinander war ursprünglich wohl kaum ein anderes als in den nächsten verwandten Sprachen. Gerade mit Hinweis hierauf ist es nur natürlich, für das Frühurlappische eine 3. Sg. Präs. vom Typ *kantaja anzunehmen, da die Form der 3. Pl. auf die Lautgestalt *kantaja-t zurückführbar ist. Man kann es als Ergebnis einer einfachen Analogie erklären, dass die urspr., dem Partizip Präs. gleiche Form der 3. Sg. verschwand. Im Singular des Imperfekts war die frühurlp. 1. und 2. Pers. *koǹnaj-ă-m bzw. *koǹnaj-ă-k aus der 3. Pers. *kondaj gebildet worden, indem man an letztgenannte - durch Vermittlung des Bindevokals ${ }^{*} \breve{a}\left(<\right.$ frühurfi. $\left.{ }^{*} e\right)$ - die betreffende Personalendung hängte. Auf das Muster dieser Reihe gestützt konnte man durch Trennung der Personalendung der 1. und 2. Pers. 
Präs. *kondam, *kondak als Form der 3. Pers. *koǹda abstrahieren. Die Entwicklung wäre also prinzipiell ähnlich gewesen wie in Südestnischen, dessen dem Verbstamm entsprechende Formen $a \bar{n} D$ 'er gibt', piḋ̈ 'er lı̈̈lt', nägè 'er sieht' gerade so erklärt worden sind, s. KETTUN Ex Virittäjä 1914 S. 145-150. Das helle a der Form *koìda ( $>\operatorname{lpN}$ guod'da), das etymologisch also nur der Auslautvokal des Stammes ist, erhielt durch Sekretion den Charakter eines Präsenssuffixes und setzte sich in vielen Stellungen durch: in der 3. Sg. Präs. der gleichsilbigen und kontrahierenden ungleichsilbigen $\breve{a}$-Stämme (z.B. *măñă $>{ }^{*}$ măna $>\mathrm{N}$ mânna 'er geht'; *perai ïă $>{ }^{*}$ pera $a \ddot{j} a>\mathrm{L}$ $p^{\dot{e} e r} \bar{k} \bar{e}$, I piěr $\bar{G} \bar{e}$ [N dagegen analogisch bir'gi statt *bier'ge] 'er behilft sich') sowie offenbar in allen singularischen Personen in den dreisilbigen $\breve{a}$-stämmigen Verben ausser in den kontrahierenden, ferner auch im Potentialis (obwohl das Potentialzeichen *ńńžă lautete), s. MSFOu 79 S. 200-202, Korhonen, a.a.O. 190-192. $\mathrm{Zu}$ diesen Verallgemeinerungen kam es schon vor dem Wandel $a>e(\varepsilon)$, der bekanntlich in den nichtersten Silben eintrat, wenn ${ }^{*} a$ in der letzten Silbe oder vor ${ }^{*} a$ bzw. ${ }^{*} \omega$ stand, z.B. frühurfi. *pesä $>$ frühurlp. *pœ̇̀a $>$ späturlp. *pase > LpN bøsse 'Nest'; frühurfi. *kantadak > frühurlp.

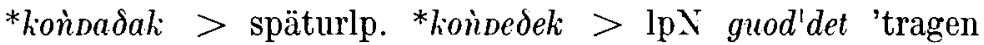
(Inf.)'; frühurfi. * parampa > frühurlp. * poramsw (der Labialrokal des Komparativsuffixes beruht wahrscheinlich auf der Einwirkung des Superlativsuffixes $\left.{ }^{*} m \omega s \sim{ }^{*} m u \dot{s} a\right)$ ) $>$ späturlp. ${ }^{*}$ porem $_{B} \omega>\operatorname{lpN}$ buoreb 'besser'.

Die stufenweise Engung des hellen $a$ (=im Frühurlp. $\left.{ }^{*} \dot{a}\right)$ zu $e$ ist - wie auch der verschiedenerseits nach der urlp. Zeit eingetretene Wandel von $e$ zu $i$ (z.B. lpN Polmak sezssi' 'Nest',

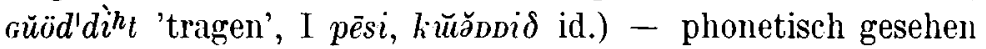
ein Schwächungsprozess, dem ein Vokal ohne semantische Wichtigkeit ausgesetzt werden konnte. Ferner kamm man für diesen Lautwandel vielleicht auch eine phonologische Motivation anführen. In einer bestimmten Phase des Frühurlappischen gab es in der zweiten und vierten Silbe nur drei Vokale: dunkles $\breve{a}\left(={ }^{*} e\right)$, helles $a\left(={ }^{*} \dot{a}\right)$ und $\omega\left(=*_{n}\right)$. In der dritten Silbe begegneten dieselben Vokale sowie ausserdem $u$ (z.B. *norămus̀ă > N nuorâmus 'der jüngste') und wahrscheinlich 
auch schon $a\left(={ }^{*} \varepsilon\right)$, z.B. ${ }^{*}$ por̀anæìa $>\mathrm{N}$ buorranabme 'Genesung'. Verglichen mit dem Vokalsystem der ersten Silbe, von dessen neun Vertretern drei vordere Vokale, zwei Mittelzungenvokale und vier hintere Vokale waren, war der Vokalismus der nichtersten Silben unharmonisch, da er nur einen Vordervokal besass, der nur unter sehr begrenzten Voraussetzungen zur Verwendung kam. Wir können somit annehmen, dass im Urlappischen dieselbe Tendenz zu wirken begann, die ganz offensichtlich die Entwicklung z.B. des ostseefinnischen und ungarischen Vokalismus prägt, nämlich das Streben nach Annäherung des Vokalismus der nichtersten Silben an den der ersten Silbe. Sie setzte sich z.B. für die zweite Silbe gerade durch den Wandel ${ }^{*} \dot{a}>{ }^{*} \varepsilon>e$ recht effektiv durch. Aus dem so entstandenen $e$ entwickelte sich schon in urlappischer Zeit noch ein anderer vorderer Vokal $i$, wenigstens (in unbetonter Stellung) vor einem silbenschliessenden $j$ bzw. mouillierten Konsonanten, z.B. 3. Sg. Imperf. *konvaj $\left({ }^{*}\right.$ kondajj) $>*^{*}$ kon${ }_{\text {DEj }}>{ }^{*}$ kondej $>{ }^{*}$ kondij $>\mathrm{N}$ güddii 'er trug'. Es ist rorläufig unsicher, ob der dem Westlappischen eigene Wandel des $\omega$ der zweiten Silbe zu $u$ vor einem $\breve{a}$ der dritten Silbe (z.B. urlp. *akkwmă $>\mathrm{N} a k^{\prime} k u m$ 'meine Grossmutter'; vgl. Nom. Sg. $\left.a k^{\prime} k o\right)$ bereits urlappisch ist. Im Ostlappischen ist diese Erscheinung unbekannt; zwar treten im Terlappischen daron vielleicht einige rudimentäre Spuren auf, die sich jedoch mittels des heute zur Verfügung stehenden Materials schwer mit Sicherheit nachweisen lassen. Jedenfalls wurde für den Vokalismus der nichtersten Silben im Urlappischen auf diese Weise schon sehr frühzeitig eine harmonische Struktur geltend: i $\underset{\varepsilon}{e} u \mid \varepsilon \dot{a} \curvearrowleft$. Als dann in einer späteren Phase des Urlappischen die Kontraktionsvokale entstanden, so glichen sie sich qualitativ den genannten nichtkontrahierten Vokalen an: von den letztgenannten blieb nur $*_{e}(=\check{a})$ ausserhalb der Kontraktionsvokale.

Unbeantwortet ist noch die Frage, warum ein helles $a$ im allgemeinen zu $e$ geworden ist im absoluten Auslaut sowie vor den offenen Vokalen $a$ und $\omega$, vor den engen Vokalen $\check{a}$ und $u$ aber erhalten blieb. Verf. hat in den MSFOu 79 S. 151-156 vermutet, dass es sich hier in erster Linie um eine (über die 
Zwischenstufe * $\ddot{a}$ verwirklichte) Palatalisierungstendenz handle, doch stehen dieser Erklärung offensichtliche Schwierigkeiten im Wege. Glaubhafter ist, dass der Wandel des $a$ (d.h. $\dot{a}$ ) letztlich durch die nachlässige Aussprache veranlasst wurde, die zu einer Hebung des Vorderteils des Zungenrückens führte, m.a.W. zur gleichzeitigen Verengung des Artikulationsgrades und zur Palatalisation. Wo nun dem $a$ ein geschlossener Vokal folgte, wollte man offenbar einen deutlichen artikulatorischen Kontrast zwischen diesen beiden Vokalen bewahren, wobei dem $a$ in dieser Stellung zweifellos mehr Artikulationsenergie zuteil wurde als wenn auch in der folgenden Silbe ein ähnlicher offener Vokal stand. Wenn die Erhaltung des a somit der sorgfältigen Artikulation zu verdanken war, ist es gut verständlich, dass es auch in der letzten Silbe erhalten bleiben konnte, wenn es, wie in den Fällen guoddam, guod'da usw., Träger einer wichtigen grammatischen Funktion geworden war.

In der 3. Sg. der o-stämmigen Verben vom Typ gill ljo, orro hat sich der abstrahierte Wortstamm als solcher erhalten. Wir haben hier also kein suffixales Element; das sekundäre Präsenszeichen $a$ konnte in diesem Stammtypus nicht Fuss fassen. Für das Ume-, Pite-, Lule- und Tornelappische würde sich der dort begegnende Typus gil'lju, orru äusserst leicht als Analogie zur entsprechenden Pluralform gil'ljuk 'sie schreien', orruk 'sie sind' erklären lassen. Das $u$ in der letztgenannten Form ist ebenfalls sekundär, aus * $\omega$ verkürzt, vgl. oben S. 109. Auf westlappischer Seite begegnen zusätzlich zu den Fällen gil'lju, gil'ljuk, gud'dik mehrere andere Konjugationsformen in unterschiedlicher Verbreitung, die den auf schnellem Sprechen u.ä. Faktoren beruhenden Wandel $e>i, \omega>u$ aufweisen. Hierher gehören im Indikativ Präsens die 1. Du. gud'di, gil' $7 j u$, die 2. Pl. -ēppit, -bēttit, im Imperativ I die 3. Sg. *gud'dis, *gil'ljus, die 2. Du. *gud'di, *gil'lju, die 3. Du. ${ }^{*} g u d d^{\prime} d i s k a \hat{,}$ * gill ljuskâ, die 2. Pl. gud'dit, gil'ljut, die 3. Pl. *gud disek, ${ }^{*}$ gil'ljusek, im Imperativ II (im Optativ) die 3. Sg. gud'dus, gil'ljus, die 1. Du. *gud'du, *gil'lju, die 3. Du. gud'duskâ, gil'ljuskâ, die 1. Pl. gud'dup, gil'ljup, die 3. Pl. gud'dusek, gil'ljusck. Problematisch wird die Frage nach der Herkunft der 3. Sg. vom Typ gil'lju dadurch, dass sie auch im Südlappischen begegnet 


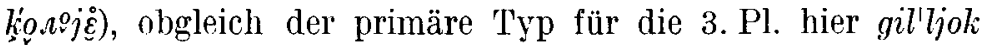
(= kia.ajuos) lautet. Entweder ist das südlappische gil lju eine relativ späte Entlehnung aus den nördlicheren Dialekten, in erster Linie aus dem Umelappischen (auf die Möglichkeit derartiger Entlehnungen ist mitunter auch in anderem Zusammenhang bei südlappischen Lauterscheinungen hingewiesen worden), oder es kam im Grenzgebiet der ursprünglichen und sekundären Typen bereits in einer früheren Phase der Sprachentwicklung zu einer Dialektvermischung, aufgrund derer beide Typen ins heutige Südlappisch gelangten, der mit $u$ in den Singular und der mit $\omega$ in den Plural.

\section{II}

Dass im Urlappischen die Endung der 3. Pl. Imperf. (Prät.) ${ }^{*} n$ lautete, ist, soweit bekannt, einmütig von den Forschern vertreten worden, nur CoLlinder macht eine Ausnahme, indem er etwas überraschend sagt, dass "in the preterite the $3 \mathrm{pl}$ ended in *-n- + vowel (lpN guid'di, Lule guod'din)", s. Comparative Grammar 306. Es bleibt unklar, warum am Ende der Endung ein Vokal anzunehmen wäre. Gut verständlich ist, dass das Auslaut- $n$ im Lulelappischen und in den meisten anderen Dialekten (vgl. Korhonen a.a.0. 338) dank seiner semantischen Funktion hat erhalten werden können. Das spurlose Verschwin-

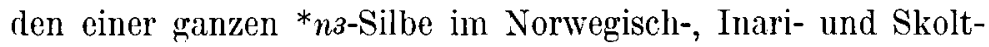
lappischen wäre dagegen eine einzigartige Erscheinung, und ausserdem ist der Typus lpI kŭŏoDi 'sie trugen' mit seinem langen Vokal der zweiten Silbe eine typische, auf eine dreisilbige Urform zurückgehende Kontraktionsform.

Es ist sehr gut möglich, dass das in Frage stehende $n$ etymologisch identisch ist mit dem $n$-Koaffix der Possessivsuffixe, das mehrere Besitze anzeigt (z.B. lpSk. Suonikylä pärnaD 'deine Söhne' < urlp. *parna-n-pă), vgl. AsbJørn Nesheim Studia Septentrionalia II 175. Andererseits ist es als Zeichen des Plurals in der Verbalflexion ganz isoliert sowohl im Lappischen als auch im gesamten finnisch-wolgaischen Sprachzweig. Werfen wir einen Blick auf die nächsten verwandten Sprachen des Lappischen, so sehen wir, dass in ihnen in der 3. Pers. Präs. 
wie auch Prät. (Imperf.) dasselbe Pluralzeichen begegnet, nämlich ${ }^{*} t$. So lautet z.B. die 3. Imperf. im Finnischen urspr. tuli-t 'sie kamen' (begegnet in der alten Sprache und in einigen Dialekten), vgl. tuleva-t 'sie kommen'. Ebenso verhält es sich in den wolgaischen Sprachen: mordw. kandoś-t 'sie trugen, brachten', vgl. kandi-t' 'sie tragen, bringen'; tscher. (dial.) $t \grave{o} \cdot l \hat{\partial}-t^{\prime}{ }^{\prime}\left(<{ }^{*} t \hat{o} \cdot l \hat{\partial}-t^{\prime}\right)$ 'sie kamen', vgl. tòे lâ-t 'sie kommen'.

Zur Vermeidung ron Homonymie kam es in der fi.-wolg. Gruppe in der Form der 3. Pl. verschiedentlich zu einer Sonderentwicklung. In einem Teil des ostseefinnischen Sprachraums wurde der Typus tulit ersetzt durch eine Form, deren Endung vat (vät) aus der Präsensflexion entlehnt wurde (tulivat), da tulit lautlich zusammenfiel mit der 2. Sg. Imperf. Im Jordwinischen ging der Form kandośt aller Wahrscheinlichkeit nach ein *kandic' voraus. Dass die Form mit dem $s$-Suffix an die Stelle des früheren Typus trat, »dürfte so zu erklären sein, dass die 3. Person (kandị) des Präsens durch den Schwund von a mit der Form des Präteritums zusammenfiel, so dass man zu einem zweiten Suffix greifen musste, das in einigen Fällen auch gebräuchlich war” (PAavo Ravila JSFOu 59,4 S. 9). Es sei noch hinzugefügt, dass *kandį ${ }^{*}$ ausser mit der 3 . Pl. Präs. auch mit der 2. Sg. Prät. homonym war (die beiden letztgenannten lauten auch in der heutigen Sprache gleich). Die ursprüngliche, mit dem Suffix *j gebildete 3. Pl. Prät. hat sich in dem enklitisch verwendeten Verb ulems erhalten, z.B. palavolt', -ult' 'sie würden küssen', vgl. J. Budesz NyK XIII 75, Ravila, a.a.O. Der Unterzeichnete vertritt die Auffassung, dass das präteritale $s$-Suffix eine Entlehnung aus der Flexion des Verneinungsverbs darstellt, vgl. MSFOu 125 S. 114. Auch im Tscheremissischen ist der primäre 'Typus tò $\cdot$ lât's nur in einem Teil der Mundarten erhalten; in einem grossen Gebiet wurde er

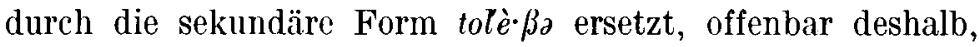
weil die in Frage stehende Form unterschieden werden sollte von der 2 . Sg. Prät. tò lât's. Die Endung $\beta$ a ist zusammengestellt worden mit dem in der verneinenden Flexion auftretenden Suffix $p: a \cdot \gamma e p$ (3. Pl. des absolut verwendeten Verneinungsverbs), $a \cdot k$ tolep 'sie kommen nicht'.

Gemeinsam für alle oben angeführten Veränderungen, die in 
der Struktur der 3. Pl. eingetreten sind, ist der Austausch der urspr. Form gegen eine andere, da erstere lautlich mit der 2. Sg. Prät. zusammenfiel, die wiederum überall in unveränderter Lautgestalt erhalten ist.

Die Annahme, dass auch das Lappische in der präteritalen Flexion ursprünglich demselben Schema wie die anderen fi.wolg. Sprachen folgte, scheint recht natürlich. Dann hätten sowohl die 3. PI. ('sie trugen') als auch die 2. Sg. ('du trugst') dem Typ *kanta-j-e-t > frühurlp. *koǹ $a-j a ̆-k$ entsprochen, der in der Gegenwartssprache fortgesetzt wird durch die Form der 2. Sg. gud'dik (= lpI kŭŏDDih, usw.). Welcher Herkunft könnte dann *koǹ $a-j a ̆-n$ sein, das zwecks Vermeidung von Homonymie an die Stelle der Form *koǹ $a-j a ̆-k$ in den Plural gelangte? Das Suffix $n$ ist in der lappischen Verbalflexion sehr allgemein. Es ist nämlich das Dualzeichen, das im Urlappischen am Ende a 1 le r Personalformen steht (im Präsens und Präteritum des Indikativs, im Potentialis, im Konditionalis und im Imperativ; z.B. 1. Du. Präs. *koǹna-ja-n, eigtl. 'zwei Träger', 3. Du. Präs. *koǹ $a-B a-n$ bzw. *-ßan, eigtl. 'zwei Tragende', 2. Du. Prät. *kond $i j-b a ̆-n)$. Rein typologisch gesehen wäre *koǹ $j \breve{a}$-n somit deutlich eine Dualform, und zwar die 3. Pers. Prät., nach genau demselben Prinzip gebildet wie die 3. Pl. Prät. *koǹba-jă- $k$, falls eine solche in der Sprache vorhanden gewesen wäre. Vielleicht wurde denn auch im Urlappischen das durch die Homonymie verursachte Problem so gelöst, dass die urspr. Dualform in den Plural überging und die neue 3. Pers. Du. Prät. durch Doppelung der $n$-Endung der urspr. Form bzw. des Dualzeichens gebildet wurde: $n+\check{a}$ (Bindevokal) $+n$. So wäre der Typus 3. Du. Prät. *konDij-năn $>1 \mathrm{pN}{ }^{*}$ güddiinâ entstanden (begegnete noch im vorigen Jahrhundert rudimentär im Dialekt von Varanger), I kŭŏDDiinn, Sk. kựppịn 'sie beide trugen'.

So liessen sich zwei Elemente der lappischen Verbalflexion in einfacher und logischer Weise erklären: die Endung $n$ der 3. Pl, die die Harmonie des Systems durchbricht, sowie die auf den ersten Blick recht eigentümliche Endung *năn der 3. Du. Die Auffassung, die vom Verf. bereits in der Zeitschrift Virittäjä 1955 S. 167-168 (vgl. Studia Fennica IX,3 S. 11-12) 
vorgebracht wurde, wonach gerade *năn von den in den lappischen Dialekten begegnenden Endungsvarianten der 3. Pers. Du. Prät. und Kond. (zu diesen s. Korhonen, a.a.O. 291-292, 293-298) am ältesten ist, wird durch die obige Erklärung weiterhin bestärkt.

ERKKI ITKONEN 